\title{
Estiramiento pasivo como opción terapéutica en paciente con hipomovilidad mandibular crónica
}

\section{Passive stretching as a therapeutic option \\ in a patient with chronic mandibular hypomobility}

\begin{abstract}
Resumen
Los trastornos mandibulares son alteraciones asociadas al dolor y disfunción de la articulación temporomandibular (ATM), a los músculos de la masticación, a la limitación del movimiento mandibular y a la presencia de un clic articular. Entre estos trastornos se puede encontrar la hipomovilidad mandibular la cual se caracteriza por la incapacidad del paciente de abrir la boca con una amplitud normal. La hipomovilidad asociada a la contractura muscular tiene como tratamiento de primera opción el estiramiento pasivo, en esta técnica el paciente tiene que abrir la boca hasta el límite máximo, este movimiento debe ser realizado durante 5 o 6 veces al día. Este reporte de caso describe una paciente de 46 años de edad con hipomovilidad mandibular por contractura muscular, se describe la sintomatología, la evaluación clínica y el tratamiento propuesto. La evolución fue favorable; en un seguimiento de 5 semanas se logró el objetivo propuesto.
\end{abstract}

Palabras clave: Diagnóstico; Contractura; Articulación temporomandibular (fuente DeCS BIREME).

\begin{abstract}
Mandibular disorders are disorders associated with the pain and dysfunction of the Temporomandibular Joint, the chewing muscles, the limitation of mandibular movement and the presence of a joint click. Among these disorders can be found mandibular hypomobility which is characterized by the patient's inability to open the mouth with a normal width. Passive stretching is the first option for hypomobility associated with muscle contraction. In this technique, the patient has to open the mouth to the maximum limit, this movement must be carried out 5 or 6 times a day. This case report describes a 46-year-old patient with Mandibular Hypomobility due to muscle contracture, the symptoms, the clinical evaluation and the proposed treatment are described. The evolution was favorable; in a 5-week follow-up, the proposed objective was achieved.
\end{abstract}

\section{Caso Clínico}

Milagros Montejo Quiroz ${ }^{1, a}$, Jorge Noriega Castañeda ${ }^{1, b}$, Rogger Bardalez Daza ${ }^{1, b}$

${ }^{1}$ Universidad Peruana de Ciencias Aplicadas, Lima, Perú.

a Cirujano Dentista, Egresado de la especialidad de

Rehabilitación Oral.

${ }^{\mathrm{b}}$ Magister en odontología.

\section{Correspondencia:}

Milagros Montejo Quiroz: milagros.montejo.quiroz@gmail.com Av. Monterrico sur 110 Block J dpto. 403 Santiago de Surco, Lima, Perú. Código postal: 15039

ORCID: 0000-0002-0597-3501

\section{Coautores:}

Jorge Noriega Castañeda: pcodjnor@upc.edu.pe ORCID: 0000-0001-5766-9539

Rogger Bardalez Daza: pcodrbar@upc.edu.pe ORCID: 0000-0001-8860-6469

\section{Editora:}

Maria Eugenia Guerrero Acevedo Universidad Nacional Mayor de San Marcos, Perú.

Conflicto de intereses: los autores declaran no tener conflicto de interés.

Fuente de financiamiento: autofinanciado

Recibido: $14 / 01 / 20$

Aceptado: $11 / 05 / 20$

Publicado: 04/08/20

Keywords: Diagnosis; Contracture; Temporomandibular joint; (source DeCS BIREME).

(C) Los autores. Este artículo es publicado por la revista Odontología Sanmarquina de la Facultad de Odontología, Universidad Nacional Mayor de San Marcos. Este es un artículo de acceso abierto, distribuido bajo los términos de la licencia Creative Commons Atribucion - No Comercia_Compartir Igual 4.0 Internacional. (http://creativecommons.org/licenses/by-nc-sa/4.0/) que permite el uso no comercial, distribución y reproducción en cualquier medio, siempre que la obra original sea debidamente citada. 


\section{Introducción}

En la actualidad, el desarrollo de la odontología requiere que el profesional de la salud se involucre en algo más que el diagnóstico y tratamiento de enfermedades dentro de la cavidad oral. Los trastornos temporomandibulares (TTM) son muy frecuentes en la población; por este motivo, los odontólogos son cada vez más responsables del reconocimiento y manejo del dolor orofacial, los trastornos de los músculos de la masticación y la musculatura asociada ${ }^{1,2}$.

Los TTM son reconocidos como uno de los temas más controvertidos en la odontología, aunque las ciencias básicas y los investigadores han llegado a un consenso. La Asociación Estadounidense de Investigación Dental (AADR), recomienda que el diagnóstico de los TTM se realice con base en los datos obtenidos de la historia clínica, examen clínico, evaluación y hallazgos de la imagen de la ATM ${ }^{1}$. La ATM está formada por la inserción del cóndilo mandibular en la fosa mandibular, los músculos de la masticación son los principales responsables del movimiento de esta articulación. Los TTM se caracterizan por un dolor agudo que puede afectar la articulación, los músculos masticatorios o los músculos de cabeza y cuello. Los factores desencadenantes pueden ser microtraumatismos, macrotraumatismos, oclusión anormal, anormalidades del disco articular, hipomovilidad mandibular crónica y hábitos parafuncionales como el bruxismo ${ }^{2,3}$.

El dolor relacionado con los TTM puede afectar en las actividades diarias, psicosociales y en la calidad de vida de la persona que padece estos trastornos ${ }^{4}$. Los estudios basados en la población muestran que el TTM afecta del $10 \%$ al $15 \%$ de los adultos, pero solo el $5 \%$ solicita tratamiento ${ }^{3}$.

En 1995, Okeson clasificó las disfunciones temporomandibulares en cuatro grandes grupos: trastornos de los músculos masticatorios, trastornos de las articulaciones temporomandibulares, hipomovilidad mandibular crónica y trastornos del crecimiento 5 .

Dentro de los trastornos de los músculos masticatorios se encuentra el dolor muscular local, este se define como la primera respuesta del tejido muscular a una co-contracción protectora continuada, siendo el trastorno doloroso agudo más habitual en la práctica clínica ${ }^{6}$.

Por otro lado, la hipomovilidad mandibular crónica es uno de los trastornos menos frecuentes, la característica principal de este trastorno es la limitación del paciente para abrir la boca con la amplitud normal. Este trastorno se subdivide en tres tipos según su etiología: anquilosis, choque coronoideo y contractura muscular ${ }^{6}$.

En el siguiente reporte de caso, se desarrollará el manejo de una paciente con dolor muscular local e hipomovilidad mandibular crónica por contractura muscular desde la fase de evaluación, examen clínico hasta la fase de tratamiento.

\section{Reporte del caso}

Paciente de sexo femenino de 46 años, procedente de Buenos Aires, Argentina acude a la consulta manifestando interés por la limitación a la apertura mandibular. Paciente firma consentimiento informado para el área de odontología y para la Especialidad de Rehabilitación Oral.

La paciente refirió no padecer ningún tipo de enfermedad sistémica, sin embargo, sufrió de neuralgia trigeminal hace 4 años aproximadamente. Inició su tratamiento odontológico; en el cual le realizaron restauraciones y exodoncias. Asimismo, recibió tratamientos endodónticos en las piezas posteroinferiores a las cuales no se le colocaron las restauraciones definitivas. La paciente indicó que presentaba dolor y dificultad para realizar la apertura mandibular, no recuerda claramente cuando empezó dicha disfunción; sin embargo, indicó que presentó episodios fuertes de estrés, los cuales desencadenaron la neuralgia trigeminal. Al examen extraoral, no presentó dolor a la palpación a nivel de la ATM; sin embargo, sí dolor del músculo masetero al realizar a la apertura bucal, la cual era de $15 \mathrm{~mm}$.

La evaluación de la ATM y de los músculos masticatorios fue realizada por la Dra. Milagros Montejo según la anamnesis y evaluación clínica descrita por Okeson en el capítulo 9 del apartado oclusión y afecciones temporomandibulares ${ }^{6}$. La paciente refirió que padecía dolores de cabeza constantes relacionado con estrés y actividad laboral. Asimismo, presentó dolores a nivel de cuello, espalda y una apertura limitada de $15 \mathrm{~mm}$ con dolor muy intenso (grado 8) según la escala de medición del dolor de Campbell ${ }^{7}$. La paciente refirió que el problema de la apertura bucal inició hace aproximadamente 4 años, cuando atravesó una situación difícil. De la misma manera, presentó dolor a nivel de los músculos maseteros el cual fue diagnosticado con dolor muscular local.

Al realizar el examen clínico de la ATM no se apreció dolor; sin embargo, al realizar la palpación de los músculos sólo se apreció dolor muy intenso (grado 8) a nivel de ambos maseteros ${ }^{7}$. En cuanto al rango y desviación de movimientos mandibulares la máxima excursión lateral derecha es de $5 \mathrm{~mm}$ y la máxima excursión de lateralidad izquierda $4 \mathrm{~mm}$ ambas asintomáticas. La apertura máxima es de $20 \mathrm{~mm}$ con dolores a nivel del músculo masetero. Después de realizado el examen para la detección de las disfunciones cráneo mandibulares se observó que la paciente padecía de hipomovilidad mandibular crónica como trastorno inflamatorio de la articulación. Se decidió evaluar las características para decidir si esta sería por anquilosis, choque coronoideo o contractura muscular. Fue descartada la primera de estas debido a que los pacientes en estos casos no refieren dolor; sin embargo, la paciente presentó dolor a la apertura y además no se observaba presencia de anquilosis fibrosa de la ATM en la radiografía panorámica (Figura 1). Por otro lado, se descartó la presencia de la hipomovilidad por choque coronoideo, ya que no cumplía las características indicadas. Finalmente, se confirmó el diagnóstico de hipomovilidad muscular por contractura muscular, 
debido a que la paciente describió antecedentes prolongados de limitación del movimiento mandibular iniciado post situación de estrés, neuralgia trigeminal y dolor muscular local. El tratamiento para esta patología fue el estiramiento pasivo, el cual consistió en el alargamiento de los músculos elevadores en donde el paciente abre la boca hasta el límite máximo del movimiento y luego realiza un estiramiento más suave, más allá de la limitación, como nos los muestra Okeson en su libro Tratamiento de oclusión y afecciones temporomandibulares (Figura 2) ${ }^{6}$. El tratamiento se realizó durante 5 semanas, en la semana 1 se recibió a la paciente con dolor y limitación a la apertura bucal de $15 \mathrm{~mm}$ (Figura 3). Se le indicó realizar técnicas de autorregulación física, en las cuales la paciente debía limitar los contactos dentales no funcionales y poner en práctica la consciencia cognitiva. Asimismo, se le indicó realizar la técnica de estiramiento pasivo, en la cual la paciente tendría que abrir la boca hasta el límite máximo del movimiento, luego debió realizar un estiramiento suave más allá de la limitación (Figura 2). Se le indicó realizar estos ejercicios cinco o seis veces a lo largo del día, con una duración de 1 o 2 segundos por cuatro veces. La paciente debía colocar los dedos entre los dientes y fomentar la apertura cuando se encontrara relajada. En la semana 2 la apertura mandibular aumentó a $20 \mathrm{~mm}$ (Figura 4), en la semana 3 a 25 $\mathrm{mm}$ (Figura 5), en la semana 4 a $33 \mathrm{~mm}$ (Figura 6) y en la semana 5 a $40 \mathrm{~mm}$ (Figura 7).

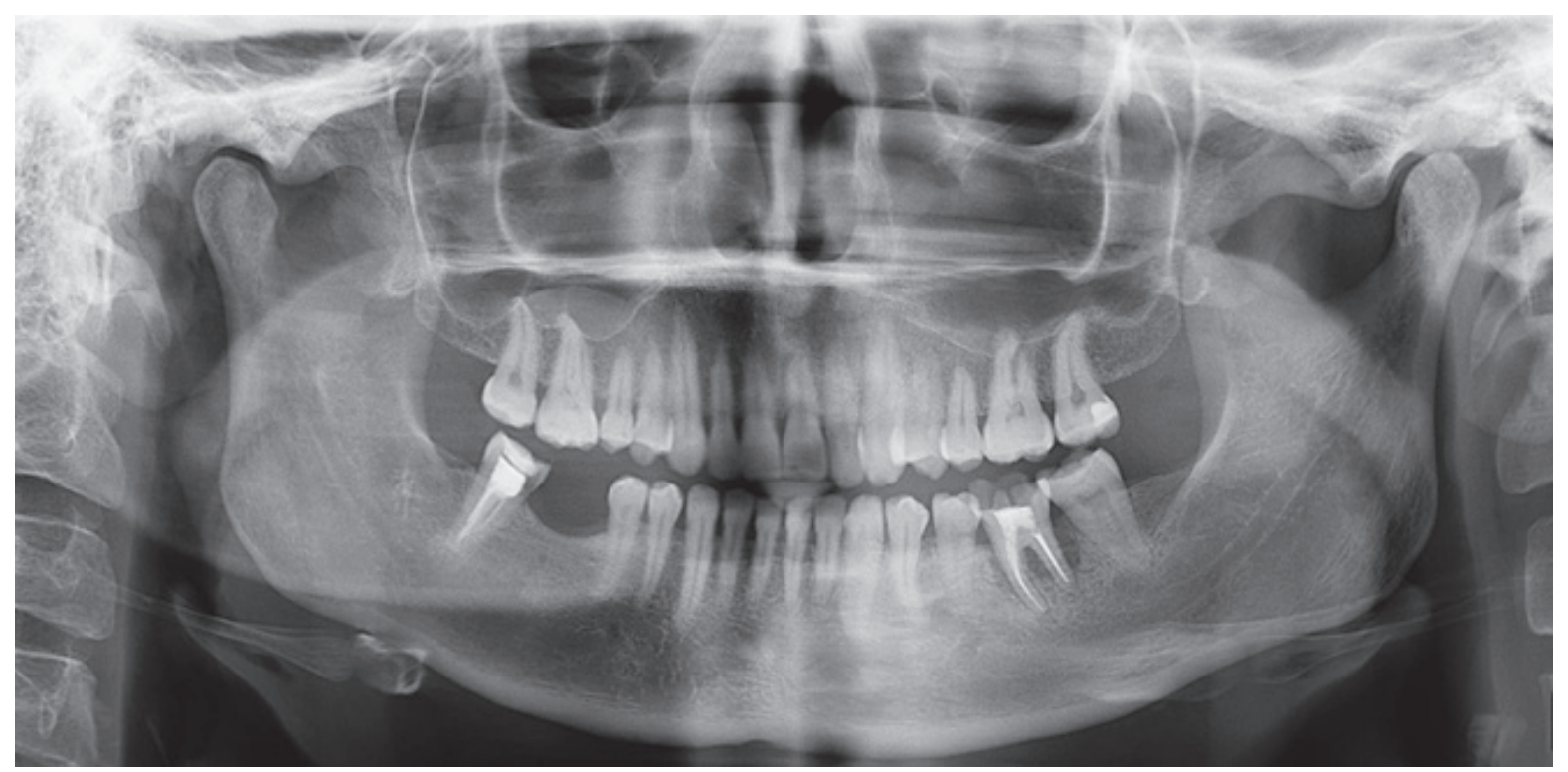

Figura 1. Radiografía panorámica sin evidencia de anquilosis fibrosa

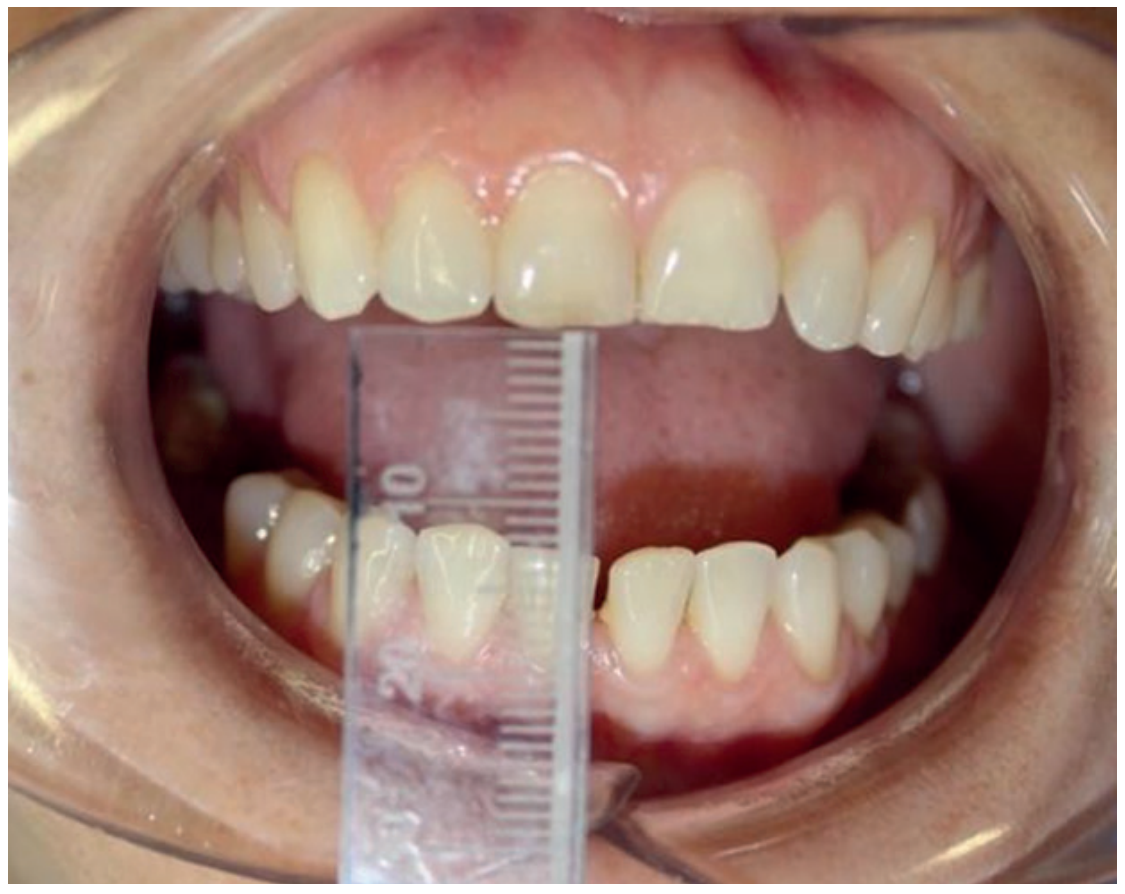

Figura 2. Fotografía a la primera semana de medición donde se registra $15 \mathrm{~mm}$ de apertura mandibular 


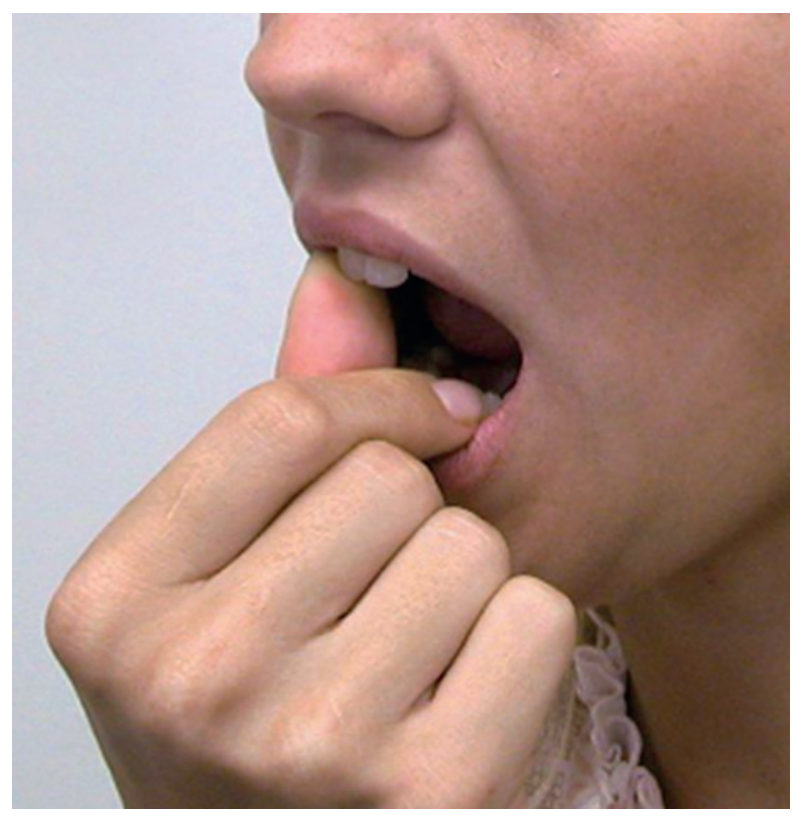

Figura 3. Técnica de estiramiento pasivo según Jeffrey P. Okeson

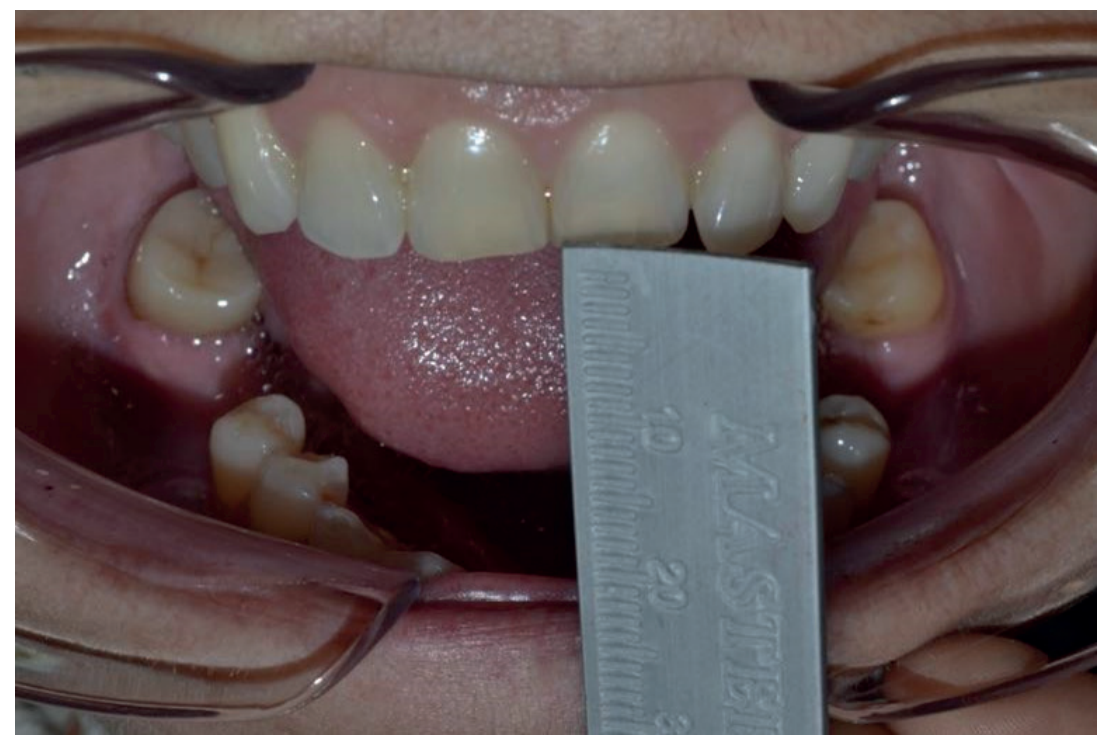

Figura 4. Fotografía a la segunda semana donde se registra un aumento de la apertura mandibular de $20 \mathrm{~mm}$

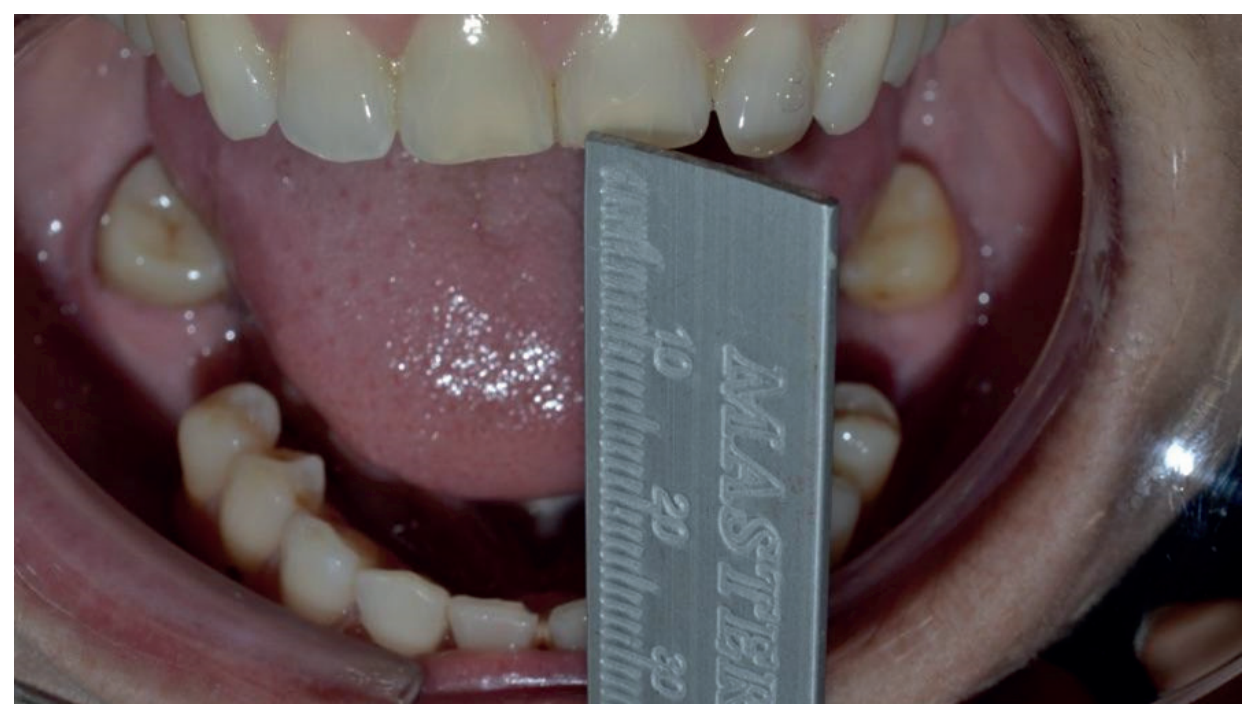

Figura 5. Fotografía a la tercera semana donde se registra un aumento de la apertura mandibular de $25 \mathrm{~mm}$ 


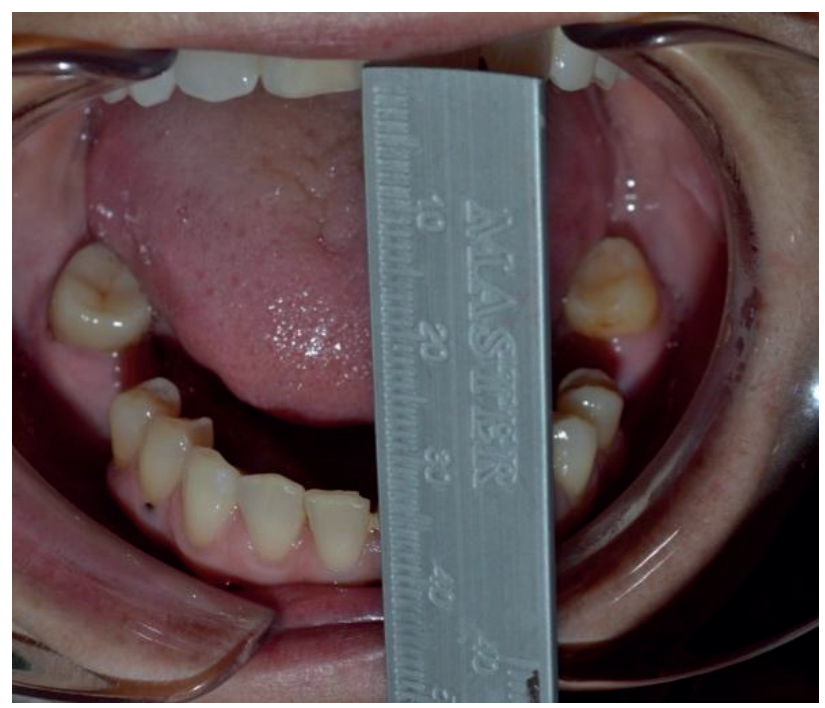

Figura 6. Fotografía a la cuarta semana donde se registra un aumento de la apertura mandibular de $33 \mathrm{~mm}$

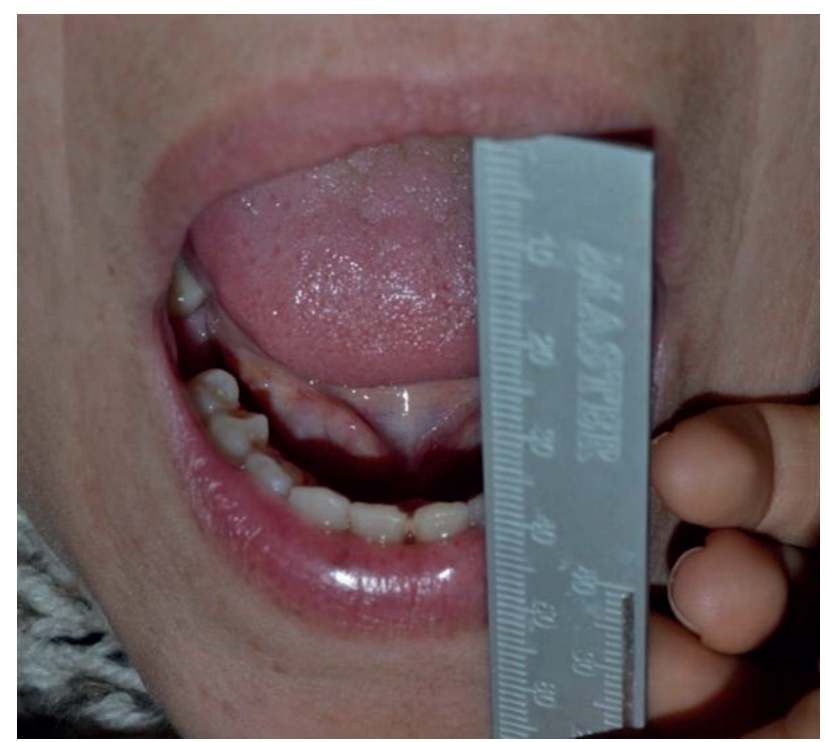

Figura 7. Fotografía a la quinta semana donde se registra un aumento de la apertura mandibular a $40 \mathrm{~mm}$

Al finalizar el tratamiento indicado se observó que la apertura mandibular mejoró significativamente, ya que al inicio era de $15 \mathrm{~mm}$ y al finalizar el tratamiento fue de $40 \mathrm{~mm}$ (Figura 7).

\section{Discusión}

La ATM una de las articulaciones más complejas del cuerpo humano, es responsable de los movimientos de apertura, cierre, retrusión, protrusión y desviaciones laterales de la mandíbula en el hueso temporal ${ }^{8}$. Por este motivo, el presente reporte tiene como principal importancia demostrar la mejor opción de tratamiento para la hipomovildad mandibular crónica, la cual se caracteriza por la incapacidad del paciente para abrir la boca con una amplitud normal. Puede estar asociada a tres factores: anquilosis, choque coronoideo o contractura muscular ${ }^{6}$.
Se sabe que los factores psicológicos desempeñan un importante papel en la etiología y persistencia del trastorno temporoandibular (TTM) 9 . Según Marpaung et al ${ }^{10}$, una forma de clasificar los diferentes tipos de TTM es dividiéndolos en dos amplias categorías: TTM relacionados con el dolor y TTM intraarticulares. En la primera categoría el dolor puede originarse a partir de las ATM, pero con mayor frecuencia los músculos masticatorios están involucrados. Por otro lado, en 1995, Okeson clasificó los TTM en cuatro grandes grupos: trastornos de los músculos masticatorios, trastornos de las articulaciones temporomandibulares, hipomovilidad mandibular crónica y trastornos del crecimiento ${ }^{6}$.

Costa $e a{ }^{11}$, resaltan que la hiperplasia por choque coronoideo es una condición rara, la cual tiene como única opción de tratamiento la cirugía, siendo la coronoidectomía intraoral la más común. Sin embargo, Okeson ${ }^{6}$ 
indica que se puede tratar con la aplicación de ultrasonidos y terapia, y recurrir a la opción quirúrgica solo en caso de deterioro grave de la función.

Según Lecas et al ${ }^{12}$, los TTM inician desde prácticas de autocuidado, tratamiento conservador, hasta llegar a cirugía. Asimismo, Okeson ${ }^{6}$, muestra la técnica de estiramiento pasivo como tratamiento definitivo para la hipomovilidad mandibular crónica por contractura muscular. En esta técnica el paciente tiene que abrir la boca hasta el límite máximo del movimiento y luego realizar un estiramiento suave más allá de la limitación. Este tratamiento debe ser suave y momentáneo para no provocar injuria en los tejidos. Este movimiento hará que el huso muscular relaje el músculo. Los pacientes deben realizar este ejercicio cinco o seis veces a lo largo del día, con una duración de 1 o 2 segundos por cuatro veces ${ }^{13,14}$. Esta fue la opción utilizada para la paciente, con la cual se obtuvieron resultados favorables.

Asimismo, según Khawaja et al ${ }^{15}$, el tratamiento de toxina botulínica tipo A (Bot-A) proporciona un alivio significativo para un tercio de los pacientes con dolor muscular. Esta terapia presenta un leve riesgo de efectos adversos. Sin embargo, Okeson ${ }^{6}$ indica iniciar por tratamientos conservadores, entre estos se encuentran las terapias de autorregulación física, aparatos de estabilización, analgésicos y termoterapia.

En este reporte se puede concluir que, a pesar de no ser un trastorno común, la hipomolividad mandibular puede ser asociada a diversos factores, en este caso se evidenció que la hipomovilidad por contractura muscular con tratamiento no invasivo tuvo una recuperación en un intervalo de 5 semanas. Como primera opción se debe aplicar los tratamientos reversibles; si se observa que estos no muestran buenos resultados, se deben utilizar los tratamientos irreversibles ${ }^{6}$.

\section{Referencias bibliográficas}

1. Aldrigue R, Sanchez A, Urban V, Pavarina A, Janaina J, Campanha N. A Survey of the Management of Patients with Temporomandibular Disorders by General Dental Practitioners in Southern Brazil. ACP. 2016;25(1):33-8. DOI: $10.1111 /$ jopr.12255.

2. Rodriguez A, Espí-López G, Langa Y. Efectividad de la terapia manual en los transtornos temporomandibulares: revisión bibliográfica. Rev Mex Med Fis Rehab. 2014;26(3-4):82-93. DOI:10.2519/jospt.2010.3257.

3. Gauer R, Semidey M. Diagnosis and Treatment of Temporomandibular Disorders. AAFP. 2015;91(6):378-86.

4. Schiffman E, Ohrbach R, Truelove E, Look J, Anderson G, Goulet J, et al. Diagnostic Criteria for Temporomandibular Disorders (DC/TMD) for Clinical and Research Applications: Recommendations of the International RDC/TMD Consortium Network and Orofacial Pain Special Interest Group. J Oral Facial Pain Headache. 2014;28(1):6-27. DOI:10.11607/jop.1151.
5. Montesinos M. Ensayo Clínico: Análisis de la eficacia del tratamiento fisioterápico en las disfunciones témporo-mandibulares para la mejora del rendimiento deportivo en saltadores de altura. [Tesis de fin de grado]. España: Universidad de La Laguna; 2016.

6. Okeson J. Tratamiento de Oclusión y Afecciones Temporomandibulres. $7^{\mathrm{a}}$ ed. Barcelona: Elseiver; 2013

7. Pardo C, Muñoz T, Chamorro C. Monitorización del dolor. Recomendaciones del gurpo de trabajo de analgesia y sedación de la SEMYCYUC. Med. Intenciva. 2006;30(8):1-7. DOI:10.1016/S02105691(06)74552-1

8. Gontijo V, Bueno K, Silva D, Alves D, Souza V. Temporomandibular dysfunction, stress and common mental disorder in university students. Acta Ortop Bras. 2016;24(6):330-3. DOI:10.1590/1413785220162406162873

9. Rokaya D, Suttagul K, Joshi S, Prakash B, Kumar P, Dixit S. An epidemiological study on the prevalence of the temporomandibular disorder and associated history and problems in Nepalese subjects. J Dent Anesth Pain Med. 2018;18(1):27-33. DOI: 10.17245/ jdapm.2018.18.1.27

10. Marpaung C, Lobbezoo F, Selms M. Temporomandibular Disorders among Dutch Adolescents: Prevalence and Biological, Psychological, and Social Risk Indicators. Pain Res Manag. 2018;2018(1):1-9. DOI: $10.1155 / 2018 / 5053709$

11. Costa Y, Porpotatti A, Stuginski-Barbosa J, Cassano D, Bonjardim L, Conti P. Coronoid Process Hyperplasia: An Unusual Cause of Mandibular Hypomobilty. Braz Dent J. 2012;23(3):252-5. DOI: 10.1590/S010364402012000300012

12. Lescas O, Hernandez M, Sosa A, Sánchez M, Ugalde-Iglesias C, Ubaldo-Reyes L, et al. Trastornos temporomandibulares: Complejo clínico que el médico general debe conocer y saber manejar. Cátedra especial "Dr. Ignacio Chávez". UNAM. 2012;55(1):1-11.

13. Ylinen J, Takala E, Nykanen M, Kautianen H, Hakkinen A, Airaksinen OV. Effects of twelve-month strength training subsequent to twelve-month stretching exercise in treatment of chronic neck pain. J Strength Cond Res. 2006;20(2):304-8. DOI: 10.1519/R-17284.1

14. McNeil C. Managment of temporomandibular disorders: Concepts and controversies. JPD. 1997;77(5):51022. DOI: $10.1016 / s 0022-3913(97) 70145-8$

15. Khawaja S, Scrivani S, Holland N, Keith D. Effectiveness, Safety, and Predictors of Response to Botulinum Toxin Type A in Refractory Masticatory Myalgia: A Retrospective Study. J. Oral Max Surgery. 2017;75(11):2307-15. DOI: $10.1016 /$ j.joms.2017.01.031 